Voix et Images

voixetimages

\title{
Vers une typologie des analepses
}

\section{Pierre Hébert}

Volume 8, numéro 1, automne 1982

\section{Madeleine Gagnon}

URI : https://id.erudit.org/iderudit/200368ar

DOI : https://doi.org/10.7202/200368ar

Aller au sommaire du numéro

\section{Éditeur(s)}

Les Presses de l'Université du Québec

\section{ISSN}

0318-9201 (imprimé)

1705-933X (numérique)

Découvrir la revue

\section{Citer cet article}

Hébert, P. (1982). Vers une typologie des analepses. Voix et Images, 8(1), 97-109. https://doi.org/10.7202/200368ar d'utilisation que vous pouvez consulter en ligne.

https://apropos.erudit.org/fr/usagers/politique-dutilisation/ 


\title{
Vers une typologie des analepses
}

\author{
par Pierre Hébert, Université de Victoria
}

Simplement constater les mutations qu'a subies la technique du retour en arrière dans le roman québécois depuis vingt ans environ porte peu à conséquence. En revanche, l'étude de l'aspect protéiforme de cette technique peut entraîner quelque mérite à la condition d'établir, après analyse de quelques récits, une typologie capable de systématiser les variantes et, par conséquent, de contribuer à l'étude diachronique de la forme du roman québécois: voilà du même coup posé le but que nous nous fixons dans cet article. À cet effet, nous avons divisé l'approche en trois volets: le rappel, d'abord, des caractéristiques traditionnelles de la technique du retour en arrière; ensuite, l'examen des transformations apportées à cette technique dans trois romans parus après 1960 (L'Aquarium, de J. Godbout, Quelqu'un pour m'écouter, de Réal Benoit et Prochain épisode, d'Hubert Aquin, publiés en 1962, 1964 et 1965). L'étude de ces trois récits permettra de faire apparaître les mutations temporelles qui nous intéressent et, de plus, elle conduira au troisième volet de notre réflexion, l'établissement d'une typologie, sorte de grille permettant une comparaison entre les diverses facettes des retours en arrière.

Nous parlons, depuis le début, de «retour en arrière». À partir de maintenant, nous préférerons le terme analepse qu'a défini et utilisé Gérard Genette'. L'étude des analepses seules, au détriment des prolepses, pose d'ailleurs un certain embarras méthodologique: nous nous sommes souvent rendu compte, à l'analyse, de la complémentarité de ces deux techniques et leur séparation, ici, doit être comprise dans l'intention d'homogénéité dont nous voulons faire bénéficier notre analyse.

\section{Les caractéristiques de l'analepse traditionnelle}

A la suite de la description théorique qu'il fait de l'analepse, Gérard Genette lui assigne une fonction canonique, ou traditionnelle: récupérer les antécédents de l'histoire. L'analepse comble une ellipse, si elle est interne, ou livre tout simplement des informations sur le passé des personnages ou sur des éléments lointains nécessaires à la compréhension de l'histoire, si elle est externe, et, dans l'un et l'autre cas, elle se lie intimement au récit qu'elle prétend éclairer en y cousant des chaînons manquants. Il va sans dire que cette fonction s'avère d'autant plus possible que les repères chronologiques 
du récit sont précis et permettent l'emboîtement de tous les morceaux. L'analepse se présente, à la limite, comme un simple désordre, dont la fonction est autant utilitaire qu'esthétique.

Outre cette fonction que leur assigne Genette, nous avons pu observer ${ }^{2}$ que la distribution des analepses dans le récit traditionnel présente une certaine prévisibilité. En effet, on a pu remarquer leur concentration au début du récit, dans le premier tiers généralement; cette distribution obéit en définitive à leur fonction, la récupération des antécédents, qui doit s'exercer le plus tôt possible.

Ces caractéristiques relatives à la fonction et à la distribution des analepses dans le récit traditionnel s'accompagnent d'une troisième mécanique probabiliste, leur présence en texte, c'est-à-dire la partie de texte qu'elles occupent par rapport au récit premier. Nous avons pu relever que cette présence est toujours faible, voire négligeable (entre $5 \%$ et $15 \%$ du texte entier, le plus souvent).

Récupération des antécédents, distribution dans le premier tiers du texte, présence minime par rapport au récit premier: nous avons pu vérifier aisément ces trois caractéristiques dans plusieurs récits de séries culturelles différentes, jusqu'en 1960, comme L'Appel de la race, Le Survenant, Poussière sur la ville et même Le Libraire, si bien qu'il semble légitime d'affirmer que cette traditionalité colore l'ensemble de la production romanesque jusqu'en 1960. Examinons à ce stade-ci les trois récits que nous avons déjà signalé dans l'introduction.

\section{L'Aquarium:}

quelques suintements nouveaux

On connaît l'étude intéressante de Robidoux et Renaud ${ }^{3}$ sur l'Aquarium, où les auteurs ne manquent pas de signaler l'intérêt de la "composition très personnelle du temps» qui, de concert avec les autres éléments du récit, crée un «roman-poème», sorte de nouveau roman. En focalisant nos propos sur les analepses, figures dominantes de ce récit, nous pouvons soulever la question suivante: à la lumière des caractéristiques traditionnelles que nous venons d'établir, qu'est-ce que L'Aquarium apporte d'original, de distinctif?

On se rappellera d'abord brièvement I'histoire de ce roman 4 : un groupe d'individus, parmi lesquels se trouve le narrateur-personnage (qui n'aura d'autre identité que «je»), est retenu dans un pays tropical par la saison des pluies, pendant que s'organise une révolution. Le seul qui ne partageait pas leur amorphisme, connu sous le signifiant de «il», s'est enlisé sans que personne du groupe ne le secoure. Cette mort à déjà eu lieu quand s'ouvre le roman, et elle obsède le narrateur. Ce que le récit présente, donc, c'est la hantise de cette mort, l'immobilisme de tous les autres personnages, et enfin. au moment où la révolution éclate, la fuite de «je» avec Andrée, l'amie de «il», qu'elle était d'ailleurs venue rejoindre. 
L'analyse nous a permis d'étaler 24 analepses, qui doivent être réparties en deux catégories. En effet, le personnage «il w fait les frais de 13 analepses, et 11 autres analepses concernent ou les autres personnages du récit, ou certains événements particuliers (la mort d'Israël, la chasse, l'ordination de Monsignor, etc.). Quant aux analepses concernant «il», elles se subdivisent elles-mêmes en deux autres classes: sa mort, et ses activités à la Casa avant cette mort. Ainsi nous avons 24 analepses, réparties ainsi:

1. 13 au sujet de «il», dont

-7 au sujet de sa mort

- 6 au sujet de ses actions à la Casa

2. 11 au sujet d'autres personnages ou d'autres événements.

En parlant du récit traditionnel et de leurs analepses, nous avons évoqué leur fonction, leur distribution et leur présence. Examinons $L$ 'Aquarium selon ces mêmes coordonnées.

La fonction des analepses dans L'Aquarium n'est pas monovalente, et c'est pourquoi nous avons préalablement distingué deux catégories, correspondant à deux classes de fonctions différentes. Les 11 analepses de la deuxième catégorie remplissent la fonction dévolue au récit traditionnel, c'est-à-dire la récupération d'antécédents. Nous apprenons l'origine de la révolution, l'arrivée des Polonais, I'histoire de Pauline, etc. Quant aux analepses concernant «il», elles exercent une autre fonction, et encore ici il faut maintenir la distinction entre celles qui relatent ses activités à la Casa, et celles qui concernent sa mort. Les activités de «il» à la Casa ne sont pas vues comme de simples antécédents, mais retentissent constamment jusqu'au récit de «je»:

S'il vivait encore, il habiterait l'appartement au-dessus du mien. Cela me rend je l'avoue un peu nerveux - il y a mêmes des journées où je crois l'entendre marcher et jurer contre Dieu et diable - ah il savait jurer avec grâce - un cri, un flot de paroles [...] (A, pp. 23-24).

De telles analepses ne représentent pas de simples événements à replacer au bon endroit dans le passé, elles ont une incidence jusque dans le présent.

Mais ce n'est pas surtout à ce niveau que l'originalité du roman nous frappe. Examinons la dernière catégorie, les analepses rapportant la mort de "il». Ces 7 analepses nous dévoilent chacune une tranche d'un même événement, les circonstances de la mort et la mort elle-même. Ces réitérations sont bien différentes d'un autre type de répétition que l'on retrouve, par exemple, dans Poussière sur la ville, d'André Langevin, où l'avertissement de Kouri est rapporté 12 fois: dans ce cas, l'événement relaté sous forme d'analepse est toujours le même. Dans l'Aquarium, la situation est tout autre: si l'événement rapporté, comme nous l'avons dit, concerne la mort de «il», il faut bien comprendre que cette histoire nous parvient en lambeaux, émiettée en quelque sorte, et que la totalité de cette histoire exige la totalité des analepses plutôt qu'une seule. II semblerait, à la lumière de cette constatation, que l'analepse traditionnelle qui se propose de récupérer un 
antécédent le fasse habituellement d'un seul coup et que, dans le cas d'une analepse qui s'affranchit de cette fonction ou qui, en tout cas, la dépasse, l'événement puisse arriver fragmenté, opérant ainsi un début de déstructuration de l'histoire.

Si, dans l'Aquarium, la fonction des analepses s'écarte quelque peu de la tradition, il n'en va pas ainsi de leur concentration ni de leur présence. A l'instar du récit traditionnel, L'Aquarium livre plus de la moitié de ses anachronies (13 sur 24) dans le premier tiers; ajoutons, pour être encore plus convaincant, que la deuxième moitié du récit n'offre que 5 analepses. Leur présence n'étonne pas plus: 18 pages environ sur 142, c'est-à-dire un peu plus de $12 \%$.

L'étude de L'Aquarium nous entraîne enfin à scruter un nouveau problème relié aux analepses: leur mode d'insertion au récit premier. Le roman traditionnel ne soulevait pas ce problème, subordonnant presque toujours l'analepse au récit premier; soit que l'analepse était clairement signalée, par une formule-type ( «ll se rappela que...»), soit qu'une indication temporelle accomplissait la rupture ( La semaine précédente... qu'un autre signal faisait fonction d'avertisseur (voir à ce sujet les astérisques dans Poussière sur la ville, p. 20). Le roman de Jacques Godbout trouble l'eau en ce qui a trait au mode d'insertion de l'anachronie, surtout en ce qui concerne les 7 analepses rapportant la mort de «il». Celles-ci sont parfois simplement coordonnées au récit premier, c'est-à-dire rattachées par un mot, une idée qui n'indique pas le passage du récit-premier à l'analepse:

Depuis deux semaines déjà qu'il pleut sans arrêt; interminable pluie, comme ces pierres qu'égrènent les femmes du pays.

- Je vous emmerde...

qu'il criait en pleurant. Je vous emmerde. Puis il est mort, et la pluie a commencé de tomber, c'est la saison ( $A$, p. 21 ; nous soulignons).

A la limite, l'analepse est simplement juxtaposée au récit et dans ces cas (pp. 22, 26, 34, p. ex.), la séquence anachronique est isolée du texte par deux interlignes (technique d'espacement qui est employée à d'autres occasions et qui ne peut être vue comme un signal d'anachronie). De clairement subordonnée au récit premier, voilà qu'avec L'Aquarium l'analepse devient coordonnée, voire simplement juxtaposée.

L'Aquarium, donc, ouvre de nouvelles voies: certaines analepses élargissent quelque peu la fonction dévolue à ces anachronies, ajoutant à la livraison d'antécédents un morcellement de l'histoire; en outre, leur mode d'insertion n'offre pas l'harmonie à laquelle nous avait habitués le récit traditionnel. Quelqu'un pour m'écouter va porter un coup décisif à ces habitudes de lecture.

\section{Quelqu'un pour m'écouter, où un narrateur fait tout pour rompre la communication}

Le récit de Réal Benoit ${ }^{5}$ se divise en trois parties inégales. La première décrit le personnage, Rémy, en train de faire sa valise, s'apprêtant à partir 
pendant que des souvenirs l'assaillent; la deuxième interrompt franchement ce récit, et le narrateur s'interroge sur son exercice d'écriture; et la troisième reprend là où la première avait laissé, c'est-à-dire Rémy qui venait tout juste de quitter et qui, à la suite d'une panne d'auto, passe la nuit chez des amis; le lendemain, suite à des images de son enfance, il se sent beaucoup mieux.

Ce bref rappel met déjà en valeur l'importance des analepses, dont nous allons maintenant faire ressortir la fonction, la distribution, la présence et le mode d'insertion au récit premier, démarche que nous avons suivie pour L'Aquarium. Livrons tout d'abord les données brutes: nous avons repéré dans le récit 28 analepses $(15,8,5$, pour les parties I, II, III).

Pour aborder la fonction des analepses, préservons cette division naturelle du roman en trois parties. La première présente donc 15 anachronies qui, toutes, remplissent une fonction classique de récupération (p. ex. la mort du fils de Rémy, pp. 13-14; la fuite du collège, p. 28; la promenade en canot, pp. 59-60). Les analepses de la deuxième partie présentent une fonction, ou plutôt des fonctions différentes: le narrateur choisit tantôt de livrer un événement banal, in-signifiant (l'histoire du pot, p. 85), tantôt des sentiments plus ou moins ancrés dans le temps (p. 96; pp. 99-100); mieux encore, il nous présente une sorte d'analepse hypothétique, c'est-à-dire ce qu'il pourrait raconter s'il poursuivait son récit; et, pour finir, une discussion (analeptique) sur l'acte même d'écrire: «[...] quelqu'un de très près de moi donc m'a dit: et quand tu te seras tout raconté, qu'est-ce que tu écriras? (O. p. 86)». Quant à la troisième partie, elle se réinstalle dans la position canonique des analepses, orientées surtout autour du fils de Rémy (pp. 114, 118, 120,127, 134). De toute évidence, c'est la deuxième partie qu'il faut considérer ici, partie où les analepses jouent un rôle nouveau, quoiqu'il se situe aussi dans le prolongement de ce à quoi L'Aquarium nous a préparés. En effet, dans L'Aquarium, les analepses rognaient I'histoire en nous la livrant émiettée; ici, elles se montrent plus audacieuses, en déployant une histoire hypothétique, que le narrateur aurait pu raconter, ou en contestant franchement cette histoire au moyen d'une interrogation sur l'acte d'écrire. Faut-il s'étonner de ce que ces nouvelles fonctions soient dévolues aux analepses?

Quant à la distribution des analepses, elle s'accomplit grosso modo d'une manière uniforme dans tout le texte. En effet, si on divise le récit en trois parties égales d'environ 43 pages chacune, on arrive à ce genre de répartition:

$$
\begin{aligned}
& \text { pp. } 9-52: 9 \text { analepses } \\
& \text { pp. } 53-96: 10 \text { analepses } \\
& \text { pp. } 96-139: 9 \text { analepses }
\end{aligned}
$$

Cette organisation s'écarte carrément du récit traditionnel, y compris L'Aquarium: il n'est plus question ici d'une distribution dans le premier tiers, ni même dans la première demie, mais plutôt d'une dissémination dans la totalité du roman.

La présence même des analepses accentue cette nouveauté formelle: le récit compte 130 pages, dont 67 analeptiques, c'est-à-dire $51 \%$ environ. 
Toutes ces remarques au sujet de la fonction, de la distribution et de la présence des analepses libèrent une réflexion sur la présence d'une forme romanesque renouvelée, du moins sur le plan où nous nous situons. Reste le mode d'insertion de ces figures: faut-il prévoir, avec l'orientation qui vient d'être donnée, une insertion harmonieuse avec le récit premier? Non, bien sûr... Un examen attentif des moyens de jonction entre l'analyse et le récit premier nous a fait prendre conscience du rôle important joué par les points de suspension. L'on peut compter, dans le récit, 284 points de suspension; de plus, nous nous sommes rendu compte que plusieurs de ceux-ci avaient pour fonction de borner une analepse. Prenons ce cas exemplaire:

Récit II ne trouvait rien à dire, rien. II avait comme une boule d'épingles dans la bouche...

Un jour, chez les prêtres, à la lecture des notes, il n'avait $\mathrm{pu}$, à son tour, dire ses résultats à voix haute comme chacun le faisait, par ordre alphabétique; le prêtre l'avait Analepse rappelé à l'ordre trois fois, rien ne sortait, rien ne pouvait sortir, il en était lui-même atterré, alors le prêtre s'était élancé de sa tribune, l'avait saisi aux cheveux, des deux mains, et l'avait projeté à genoux... Rémy avait tout juste eu le temps de crier sa note

Récit

Premier

du mois... ce soir il n'allait pas crier [...] (0. p. 81)

Étant donné que le récit offre 28 analepses, et que chacune peut être ouverte et/ou fermée par des points de suspension, nous avons un maximum de 56 cas de ce type de jonction au récit premier:

\section{Parties du récit Nombre d'analepses}

Nombre de points de suspension fermant et/ou ouvrant l'analepse

\begin{tabular}{rrrr}
\hline II & 15 & $21 / 30$ & $(70 \%)$ \\
II & 8 & $14 / 16$ & $(87 \%)$ \\
& 5 & $3 / 10$ & $(30 \%)$ \\
& 28 & $38 / 56$ & $(68 \%)$ \\
\hline
\end{tabular}

Cette approche de l'insertion des analepses réclame deux observations: sur la signification même des points de suspension comme mode d'insertion, et sur la signification de la variation de distribution, dans les trois parties, de cette organisation.

La signification des points de suspension signale une antinomie quasi irréductible. D'une part, ces signes de ponctuation semblent jouer le rôle, par leur récurrence, de signal explicite d'une analepse prochaine, ou de sa fin et, sous cet angle, en harmonisant l'anachronie au récit, accomplit à l'aide d'un nouveau moyen ce que le récit traditionnel réussissait à l'aide de signaux que nous avons déjà mentionnés dans nos propos sur L'Aquarium; d'autre 
part, la nature même des points de suspension établit un hiatus entre l'analepse et le récit premier. En effet, ces signes de ponctuation indiquent «que l'expression de la pensée reste incomplète pour quelque raison d'ordre affectif ou autre ${ }^{6}$ : ce qu'il faut entendre par là, c'est que le discours narratif dévie vers le discours seul, c'est-à-dire l'émotivité (au sens jakobsonien) du narrateur. Cette «incursion dans l'implicite» (Bachelard) est responsable d'une rupture de communication narrative. Telle est donc la nature du paradoxe que nous avons présenté: deux signaux qui, explicitant une structure analeptique, sont eux-mêmes déviation vers l'implicite. Ainsi réussissent-ils à la fois à lier et à rompre.

A la lumière de ce dernier paragraphe, insufflons un sens à la distribution de ce mode d'insertion en vertu des trois parties du roman. On y perçoit clairement trois mouvements différents, la partie médiane étant celle où les analyses sont à la fois les plus encadrées et les plus lâches par rapport au récit premier. Quand nous avons traité de la fonction des analepses, nous avons mis l'accent sur cette même deuxième partie, où l'histoire était menacée, voire grugée par les anachronies. Leur mode d'insertion témoigne dans le même sens, et signale l'importance qu'il faut attacher aux points de suspension comme signaux de disjonction : plus que jamais la partie centrale du roman offre un visage nouveau, différent du récit traditionnel. À la limite, Quelqu'un pour $m$ 'écouter présente trois récits dans un ou, mieux, trois types de récits: la première partie prend quelques distances par rapport au récit classique, la deuxième s'en détache nettement et la troisième, au contraire, épouse les contours du récit traditionnel.

Quelqu'un pour m'écouter, au titre révélateur, pose donc des problèmes de communication, c'est-à-dire de liaison entre l'émetteur et le récepteur, quant à la possibilité de livrer une histoire, en particulier dans la deuxième partie, tant par la fonction que par l'insertion des analepses. Prochain épisode poussera plus loin ces premiers efforts d'émancipation et de transgression.

\section{Prochain épisode: \\ la transgression des catégories}

Prochain épisode ${ }^{7}$, on le sait, rapporte l'histoire d'un révolutionnaire incarcéré, «je», attablé pour écrire un roman d'espionnage afin de ponctuer son immobilité, son ennui, voire sa dépression. Ce roman policier, difficilement construit et maintes fois entrecoupé par le récit de prison, raconte à son tour I'histoire d'un “je», vraisemblablement le même que le «je» incarcéré, poursuivant un certain de Heutz, à la demande d'une femme, K. "Je" a 24 heures pour tuer de Heutz et rejoindre K. à I'hôtel d'Angleterre. Il échouera, ne retrouvera pas $K$. et n'arrivera pas vraiment à achever son récit.

Fonction, distribution, présence et insertion des analepses doivent d'abord être précédées d'une réflexion décisive: qu'est-ce qui, dans Prochain épisode, est analepse? La réponse n'est pas si simple, et il nous semble d'abord nécessaire d'identifier les récits présents dans le texte. En effet, 
il faut, croyons-nous, considérer la situation de «je» en prison, ses angoisses et son projet d'écrire comme le récit premier (R1). Ce récit offre tous les éléments fonctionnels pour fonder une histoire, c'est-à-dire, selon la définition de Propp reprise par Bremond, une succession d'événements, d'intérêt humain, présentés dans l'unité d'une même action, figurable tout au moins en séquences (élémentaires) d'amélioration et de dégradation. Ainsi, le personnage «je», voulant «peupler son vide» $(P E$, p. 3$)$ et fuir l'ennui, se dit préoccupé par un seul problème: comment s'y prendre pour écrire un roman d'espionnage? Ce récit policier, R2, représentera pour le personnage cette façon d'échapper à la décomposition intérieure et fonctionnellement, sera un moyen d'éviter la «dégradation» dont le processus est actualisé par R1, et l'agencement fonctionnel des deux récits offre le cas qu'a décrit Bremond: "on peut considérer que l'échec d'un processus d'amélioration ou de dégradation en cours résulte de l'insertion d'un processus inverse qui l'empêche d'aboutir à son terme normal ». Prochain épisode offre cette insertion d'un processus inverse, à cette différence près que celui-ci aussi échouera:

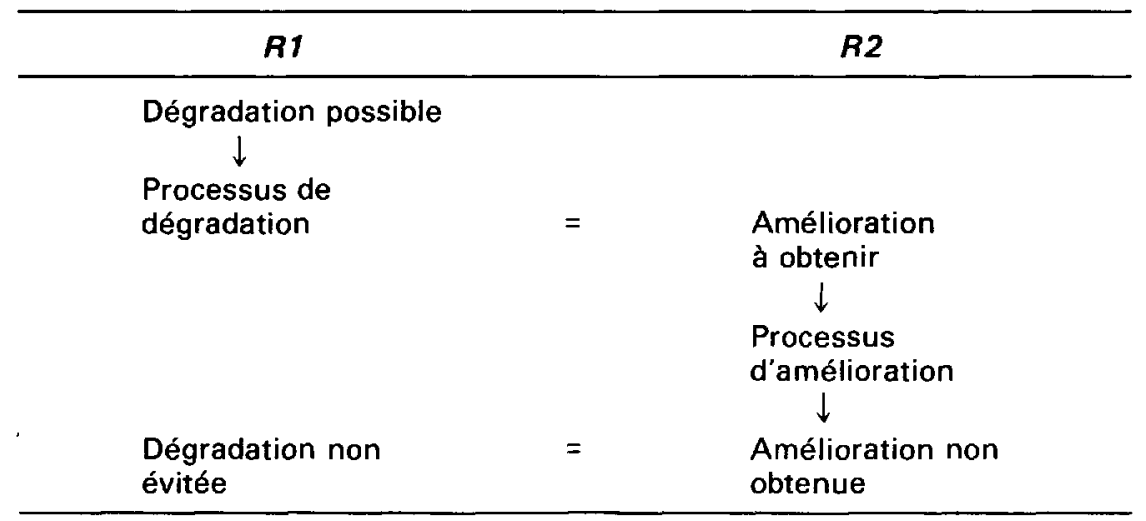

De plus, le récit d'espionnage $\mathrm{R} 2$ apparaît comme un métarécit, c'est-à-dire un récit dont le narrateur se trouve à un récit de niveau inférieur, au R1 en l'occurrence. Ainsi non seulement le R2 est-il lié au R1 fonctionnellement, c'est-à-dire comme séquence enclavée, mais en plus son narrateur est le même que celui du récit premier: «je" raconte un récit, le R1, dans lequel il raconte un autre récit, $R 2$.

Ces considérations nous ont-elles éloigné des analepses? Pas du tout, si nous reprenons notre première question: qu'est-ce qui, dans Prochain épisode, est analepse? Bien sûr, le R1 présente «ses» analepses: la promenade avec la femme aimée (p. 23), l'insurrection ratée qui conduit à la prison (p. 14), etc. Mais c'est le statut du récit (métadiégétique) d'espionnage qui nous intéresse davantage sur ce plan car il peut, tout entier, être vu comme analeptique, à la condition sine qua non de bien comprendre que, récit spéculaire, il reproduit sur un autre plan la quête révolutionnaire tentée par «je» avant son incarcération, et que cette qualité lui vaut d'être un long retour en arrière: «[...] je 
n'imagine rien de mieux que de continuer d'écrire sur cette feuille et plonger sans espoir dans le lac fantôme qui $m$ 'inonde. Descendre mot à mot dans ma fosse à souvenirs [...]» $\langle P E$, p. 15). De là émane la déroute de lecture qu'engendre le roman: l'axe du déroulement du roman d'espionnage doit être lu dans une perspective d'antériorité au récit premier: «Quelque chose me dit qu'un modèle antérieur plonge mon improvisation dans une forme atavique $[\ldots]$. Le geste futur me connaît depuis longtemps (PÉ, p. 73).» Un personnage en train "d'écrire [ses] mémoires" (p. 16) en les déguisant en roman d'espionnage métadiégétique et analeptique: cette situation devait être clairement établie avant l'étude des analepses elles-mêmes.

L'on voit maintenant jusqu'à quel point la fonction des analepses est modifiée, tout en ne l'étant pas: les anachronies récupèrent des antécédents, certes, mais sous un «mode» de transformation, et obligent au déchiffrement plutôt que de projeter la clarté; en plus, elles agissent sur le récit premier, sur le présent du narrateur en train d'écrire cette histoire pour éviter la dépréssion. Elles ont à la fois une fonction traditionnelle et nouvelle. En reprenant mutadis mutandis les mots de Benveniste, on pourrait dire que l'analepse traditionnelle doit être reconnue, c'est-à-dire identifiée comme telle et placée à l'intérieur des autres éléments du récit; dans le cas du récit d'Aquin. l'analepse doit être reconnue, certes, mais aussi comprise, en tant que mode de signifiance, de production d'un sens.

Dès lors que l'on considère le R2 comme analeptique, la distribution et la présence des anachronies se trouvent affectées. Les analepses, en effet, sont alors présentes partout; l'on pourrait même dire que plus le récit avance. plus elles s'imposent. Le premier chapitre établit les bases du R2, lequel commence péniblement au deuxième chapitre, et occupe le troisième presque tout entier; le récit continuera de présenter cette présence massive du R2, entrecoupé de séquences, brèves ou longues (cf. chapitre dix) selon le cas, du récit premier, jusqu'à la mixtion des deux récits à la fin. Il s'ensuit que la présence des analepses est également impressionnante: 82 pages sur 145 , $56 \%$ environ, proportion légèrement supérieure à Quelqu'un pour m'écouter.

Reste l'insertion des séquences analeptiques, où le récit (contrairement à toute attente peut-être?) n'offre rien de caractéristique. Les liaisons entre le R2 (analeptique) et le R1 s'établissent harmonieusement, en général. Parfois, une indication chronologique d'antériorité, symbolique certes, ouvre I'analepse: «Entre le 26 juillet cubain et la nuit lyrique du 4 août [ ... ] (PÉ, p. 19).» Souvent, les répères se feront plus clairs quant au caractère analeptique de la séquence, mais moins précis chronologiquement: «Et me voici à nouveau dans cette nuit antérieure $[\ldots](P E$, p. 19)»; «je m'égare dans ce procès-verbal. Je dérape dans les lacets du souvenir, comme je n'ai pas cessé de déraper avec ma Volvo $[\ldots](P E$, p. 33)." Enfin, la fusion des deux récits s'accomplit en toutes lettres:

C'est pouquoi sans doute, chaque fois que je prends mon élan dans ce récit décomposé, je perds aussitôt la raison de continuer et ne puis m'empêcher de considérer la futilité de ma course écrite dans l'ombre 
des Mosses et du Tornettaz, quand je songe que je suis emprisonné dans une cage irréfutable. [...] Et j'envoie mon délégué de pouvoir en Volvo dans le col des Mosses, je l'aide à se rendre sans accrochage au palier supérieur des Mosses et je lui fais dévaler l'autre versant de la montagne $[\ldots](P E$, p. 35).

Ensuite, le narrateur poursuit avec son récit d'espionnage.

Toutes ces considérations que nous avons récoltées sur les analepses doivent maintenant être orientées vers l'établissement de notre véritable objectif, une typologie capable de rendre compte de l'aspect protéiforme des rétrospections.

\section{Pour conclure:}

une typologie de classement et la mesure de l'originalité

Tout au long de cet article, nous avons comparé diverses analepses, si bien que le plus important est peut-être le moyen de comparaison lui-même. Aussi désirons-nous maintenant organiser toutes les remarques disséminées dans notre texte et former ainsi une typologie des analepses, qui pourrait inclure les paramètres suivants:

1. Distribution: en divisant le récit en trois parties égales, montrer dans quelle partie se trouve le plus grand nombre d'analepses.

2. Présence: elliptiquement, ce mot désigne "l'importance de la présence (c'est-à-dire la longueur de texte) occupée par les analepses par rapport au récit premier". Ce taux de présence, dans notre tableau, sera estimé en pourcentage.

3. Fonction: nous placerons en troisième lieu la fonction, après le recueil des «données numériques". L'analepse a, presque toujours (sinon toujours) à récupérer un antécédent: tout se situe dans la manière dont s'exerce cette récupération. L'antécédent peut être récupéré de manière unique, c'est-à-dire d'un seul coup (type qu'on finit toujours par retrouver dans un récit) ou, le cas échéant, de manière répétitive; répété, l'antécédent peut être donné en entier dans chaque réitération, comme c'est le cas pour l'avertissement de Kouri dans Poussière sur la ville; fragmenté, l'antécédent sera alors connu partiellement à chaque réitération, comme la mort de «il»dans L'Aquarium.

4. Modalité: le mode désignant toute «régulation de l'information narrative», l'analyse de divers récits nous a appris que le contenu narratif des analepses peut être livré de deux façons:

a) objectivement, c'est-à-dire le cas où les événements sont relatés tels qu'ils sont censés être arrivés;

b) subjectivement, c'est-à-dire le cas où l'événement en lui-même a fait l'objet d'une intervention du narrateur 
(ou du narrateur-personnage). Cette approche subjective de l'analepse offre elle aussi plusieurs variantes:

- I'hypothèse: le narrateur rapporte l'événement tel qu'il le suppose être arrivé;

- la transformation: le narrateur livre l'événement d'une manière tout autre qu'il s'est "réellement" produit;

- l'illusion, où le narrateur relate un événement sous des allures de rétrospection, mais un événement qui, en vérité, ne s'est jamais produit.

5. Insertion: l'anachronie rétrospective peut être rattachée au récit premier par une solution de continuité, c'est-à-dire une liaison quelconque qui peut présenter, entre le récit premier et l'analepse, un rapport ou bien de subordination, ou bien de coordination. Si ce rapport de subordination est créé par une phrase du type "Je me rappelle...", on le dira explicite; si le rapport de subordination s'exerce par un rapport plus flou (soit un répère temporel, du type «la veille...», soit un double interligne, comme dans L'Aquarium, ou des astérisques, comme dans Poussière sur la ville), on le dira implicite. Quant au rapport de coordination entre le récit et l'analepse, il se fait à l'aide d'un objet, d'um mot qui assure la jonction (voir la pluie, exemple que nous avons donné dans L'Aquarium).

L'anachronie rétrospective peut s'établir par discontinuité par rapport au récit d'où elle part. Dans ce cas, elle peut être simplement juxtaposée au récit ou, à la limite, établir à l'égard de celui-ci une rupture (comme dans Quelqu'un pour m'écouter).

On peut regrouper toutes ces données sur les analepses dans le tableau suivant: 


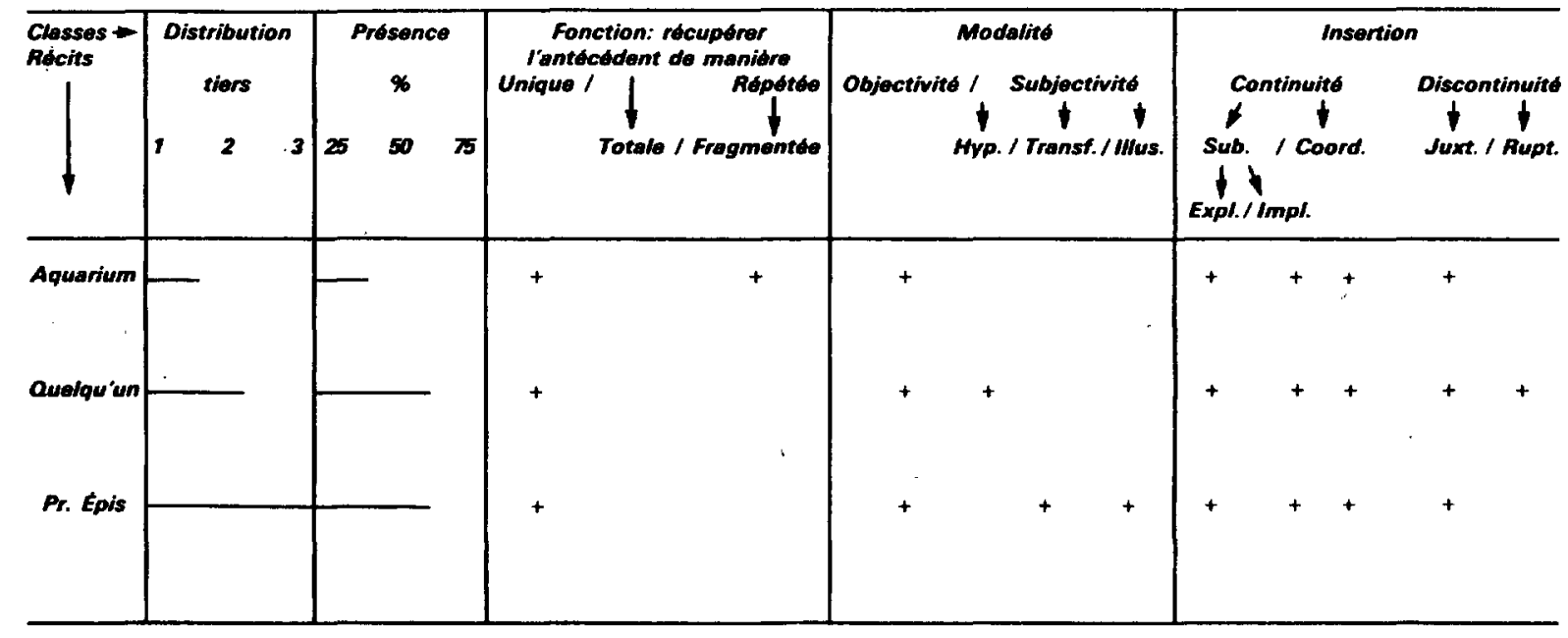


Pour terminer, nous voulons faire apparaître le rôle d'une telle typologie dans la recherche littéraire et, ainsi, démontrer l'enjeu de notre recherche.

Il est aujourd'hui convenu de distinguer trois niveaux dans la communication littéraire ${ }^{8}$ : le système, lieu des lois du récit, lois universelles ou, au moins, trans-historiques; la norme, représentée par les contraintes historiques (sociales, idéologiques, etc.) qui balisent l'utilisation du système; l'usage enfin, c'est-à-dire l'exercice particulier du système sous l'égide de la norme. En conséquence, la sémiotique littéraire exige que le chercheur résolve trois problèmes: l'étude du système, la mise à jour de la norme et la description précise des usages.

Dans cet article, nous avons isolé le troisième de ces champs de recherche, l'usage, en prenant tel quel un élément du système, l'analepse, dont nous avons étudié diverses réalisations possibles, à une époque donnée du roman québécois. Notre typologie a donc visé à établir l'originalité de trois récits, pris dans une série romanesque homogène. Or, pareille enquête présente aussi des limites évidentes, à deux des niveaux que nous avons signalés, l'usage et la norme.

En ce qui a trait à l'usage, notre enquête est limitée: il eût été possible - au delà des cadres d'un article - non seulement d'enrichir le corpus de la série culturelle que nous avons examinée, mais aussi d'étudier une autre série culturelle, par exemple le roman psychologique des années 50 , et de comparer les usages obtenus. Et sur le plan de la norme, notre recherche est sciemment muette. En effet, nous n'avons établi aucun rapport entre les usages, dont nous avons offert une typologie, et les contraintes particulières qui les ont entraînés.

Notre contribution revient donc à avoir fourni une radiographie d'un élément du système, à un moment donné de l'histoire. Et cette recherche puisque le mot histoire vient d'être écrit - trouverait tout son sens dans cette conjoncture plénière et simultanée de l'intemporel (le système), de l'historique (la norme) et de l'individuel (l'usage).

1. Gérard Genette, Figures III, Paris, Seuil, 1972, p. 90 sq.

2. Voir à ce sujet notre thèse, Figures, temporalité et forme du discours narratif, Doctorat Université Laval, 1977.

3. Réjean Robidoux et André Renaud, Le Roman canadien-français du vingtième siècle, éd. de l'Université d'Ottawa, 1966.

4. Jacques Godbout. L'Aquarium, Paris, Seuil, 1962 (désormais $A$ dans le texte).

5. Réal Benoit, Quelqu'un pour m'écouter, Montréal, CLF, 1968 (désormais $Q$ dans le texte).

6. Maurice Grevisse, Le Bon Usage, éd. Duculot, p. 974.

7. Hubert Aquin, Prochain épisode, Ottawa, éd. du Renouveau Pédagogique, 1969. (désormais $P E$ dans le texte).

8. Voir à ce sujet Études Littéraires, vol. 14, n 1, avril 1981, p. 9 sq. 